\title{
Reconstruction of Cerebrospinal Fluid Flow in the Third Ventricle Based on MRI Data
}

\author{
Vartan Kurtcuoglu ${ }^{1}$, Michaela Soellinger ${ }^{2}$, Paul Summers ${ }^{3}$, Kevin Boomsma ${ }^{1}$, \\ Dimos Poulikakos ${ }^{1}$, Peter Boesiger ${ }^{2}$, and Yiannis Ventikos ${ }^{4}$ \\ ${ }^{1}$ Laboratory of Thermodynamics in Emerging Technologies, \\ Department of Mechanical and Process Engineering, ETH Zurich, Switzerland \\ Vartan.Kurtcuogludethz.ch \\ ${ }^{2}$ Institute for Biomedical Engineering, \\ University of Zurich and ETH Zurich, Switzerland \\ ${ }^{3}$ Department of Neuroradiology, Nuffield Department of Surgery, \\ University of Oxford, UK \\ ${ }^{4}$ Fluidics and Biocomplexity Group and Institute of Biomedical Engineering, \\ Department of Engineering Science, University of Oxford, UK
}

\begin{abstract}
A finite-volume model of the cerebrospinal fluid (CSF) system encompassing the third ventricle and the aqueduct of Sylvius was used to reconstruct CSF velocity and pressure fields based on MRI data. The flow domain geometry was obtained through segmentation of MRI brain anatomy scans. The movement of the domain walls was interpolated from brain motion MRI scans. A constant pressure boundary condition (BC) was specified at the foramina of Monro. A transient velocity $\mathrm{BC}$ reconstructed from velocimetric MRI scans was employed at the inferior end of the aqueduct of Sylvius. It could be shown that a combination of MRI scans and computational fluid dynamics (CFD) simulation can be used to reconstruct the flow field in the third ventricle. Pre-interventional knowledge of patient-specific CSF flow has the potential to improve neurosurgical interventions such as shunt placement in case of hydrocephalus.
\end{abstract}

\section{Introduction}

The cerebrospinal fluid (CSF) is contained within and surrounds the brain and spinal cord [1]. It suspends the brain through its buoyancy force and protects it from impact on the cranial vault walls in cases of sudden head motion. The CSF further serves as an intermediary between blood and nervous tissue, providing the latter with nutrients and removing waste products.

CSF is produced in the choroid plexuses of the brain and is drained mainly through the superior sagittal sinus. A periodic pulsatile motion governed by the cardiac cycle is superimposed upon the steady flow caused by the CSF production. Within the skull, the cerebrospinal fluid is contained in the ventricles and the subarachnoid space. The ventricles are four cavities interconnected by pathways (see Fig. 1).

In contrast to the cardiovascular system, computational representations of the cerebrospinal fluid space are still in their infancy, despite the first attempts at such models being made as early as 1969 [2]. Since 1999, a series of models based on CFD 
and numerical structural mechanics have been published, all of which rely on either or both extensively simplified domain geometry and boundary conditions [3-6]. The finite-volume model presented here takes into account the wall motion of the third ventricle and aqueduct of Sylvius in feet-head direction based on MRI measurements. The domain geometry is obtained using anatomic MRI scans. The boundary condition at the inferior end of the aqueduct of Sylvius is implemented by reconstruction of a Womersley velocity profile based on MRI velocimetry. All of these features are novel.
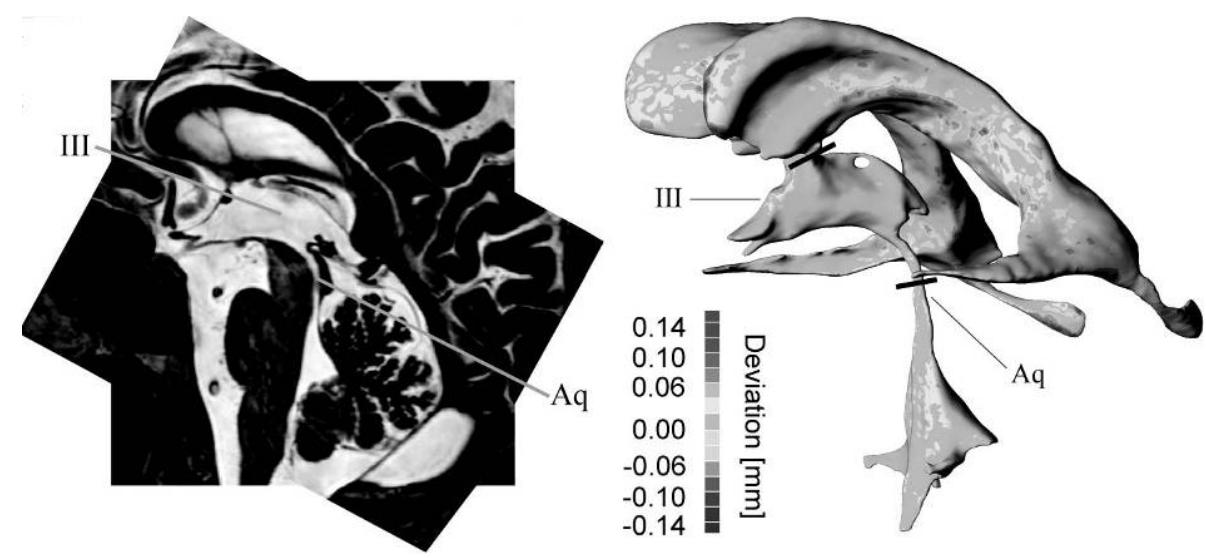

Fig. 1. Left: Composite of two registered slices of MRI anatomy scans of the ventricular system. Right: Rendering of the segmented ventricular system after conversion to NURBS surfaces. The color coding indicates the deviation between the original voxel-based segmentation and the NURBS representation. The bars indicate the boundaries of the system treated in this paper, encompassing the third ventricle (III) and aqueduct of Sylvius (Aq).

\section{Methods}

\subsection{Acquisition and Processing of Anatomy Data}

Structural MR imaging to define the ventricle and aqueduct boundaries consisted of a strongly T2-weighted, 3D, turbo spin-echo sequence [7] performed on a 27 year old healthy male volunteer, on a high-field clinical imaging system (Achieva 3T, Philips Medical Systems, Best, The Netherlands). The imaging parameters were: repetition time $(\mathrm{TR})=2325 \mathrm{~ms}$, echo time $(\mathrm{TE})=160 \mathrm{~ms}$, number of signal averages $=1$ and an echo train length of 36 echoes. The reconstructed spatial resolution was $0.22 \times 0.22$ x $0.4 \mathrm{~mm}$.

To accommodate for the slight anisotropy in the spatial resolution of imaging data, the sequence was performed three times, each acquisition in a different cardinal orientation. These three data sets were aligned by minimizing the Euclidean distance between similar gray values. The registration was further enhanced using a quasiNewton optimizer [8] and the datasets were merged using Lanczos interpolation [9]. The third ventricle and the aqueduct of Sylvius were manually segmented and the 
obtained voxel-based 3D representation was converted to NURBS surfaces (nonuniform rational B-spline). This approach ensured adequate smoothing of the domain surface which is necessary for the generation of a high-quality computational grid.

\subsection{Acquisition and Processing of Brain Motion Data}

For measurement of the brain tissue displacement, the aforementioned volunteer was scanned with a complementary spatial magnetization modulation (CSPAMM) [10] prepared sequence in a mid-sagittal slice using a tag distance of $2 \mathrm{~mm}$. The scans were performed on a whole-body MRI system (Intera 1.5T, Philips Medical Systems, Best, The Netherlands). Separate tag line preparation was performed in two orthogonal in-plane directions (cranio-caudal and anterior-posterior). The number of cardiac phases was chosen such that at least $120 \%$ of the R-R interval could be covered to allow indirect validation of the tracking error. Reproducibility of the measurements was assessed by two subsequent measurements on the same volunteer. After tag preparation, the data were acquired with a turbo field echo [7] sequence with a turbo factor of 3 and TR/TE: $8.13 / 3.8 \mathrm{~ms}$. The spatial resolution was approximately $1 \mathrm{~mm}$ and the temporal resolution $60 \mathrm{~ms}$. Cranio-caudal displacement was finally quantified using harmonic phase (HARP) [11] post-processing with a shutter width of 24 pixels which yielded a HARP tracking resolution of about $5.3 \mathrm{~mm}$ [12]. Subject to the limits of resolution of the data acquired, displacements in the anterior-posterior direction were too small to be resolved. Using least-squares error minimization, a fourth order Fourier series was fit to the time-dependent displacement data of each measured brain location. The displacement of the third ventricle and aqueduct boundaries were approximated from the measured locations using thin-plate splines.

\subsection{Acquisition and Processing of CSF Flow Velocity Data}

A standard phase contrast velocity mapping sequence [7] was also used on the same volunteer. The measured slices were planned perpendicular to the aqueduct of Sylvius and carried out in its caudal aspect where the diameter is larger than in the cranial section. The data were acquired with a turbo field echo (turbo factor $=3$ ) read-out and the spatial scan resolution was set to $288 \times 216$ (reconstruction to $512 \times 512$, covering a field of view of $250 \times 211 \times 5 \mathrm{~mm}$ ). As maximum velocity values in the range of 5 $\mathrm{cm} / \mathrm{s}$ [13] were expected, the encoding velocity was set to $7 \mathrm{~cm} / \mathrm{s}$ in order to avoid phase wraps. A total of 40 cardiac phases were generated with a temporal resolution of $26 \mathrm{~ms}$. Three signal averages were used to improve the signal-to-noise ratio. As both the spatial and temporal resolution of the velocimetric MR data are lower than what is needed for accurate CFD calculations, the algebraic solution of the flow field at the inferior end of the aqueduct was reconstructed from the measured data using the theory of pulsatile flow in a pipe [14].

For this purpose, the aqueduct was regarded as a circular cylinder and the flow at its outlet section was assumed to be fully developed. The former is a plausible simplification, as the shape of the aqueduct is indeed approximately cylindrical, albeit with variable radius. The plausibility of the latter assumption can be verified as follows: The maximum Reynolds number (Re) and minimum Womersley parameter $(\alpha)$ for the flow in the aqueduct can be calculated from the velocimetric MR data, 


$$
\operatorname{Re}_{\text {max }}=\frac{u_{\max } D}{v}, \quad \alpha_{\min }=\frac{D}{2} \sqrt{\frac{2 \pi f_{\min }}{v}},
$$

where $\mathrm{u}_{\max }$ is the highest axial velocity - both locally and temporally - at the inferior end of the aqueduct of Sylvius, D is the diameter of the aforementioned cylinder, $v$ is kinematic viscosity and $\mathrm{f}_{\min }$ is the cardiac cycle base frequency. In the case at hand, $\mathrm{Re}_{\max }$ is 70.9 and $\alpha_{\min }$ is 2.9 , which results in a hydrodynamic entrance length acceptably shorter than the length of the aqueduct $[15,16]$. Consequently, the flow field at the inferior end of the aqueduct can be regarded as being fully developed. Assuming a harmonic driving pressure gradient, the velocity field can be calculated as

$$
u(r, t)=\frac{i k R^{2}}{\mu \alpha^{2}}\left(1-J_{0}\left(A \frac{r}{R}\right)\left(J_{0}(A)\right)^{-1}\right) e^{i \omega t}, \quad A=\left(\frac{i-1}{\sqrt{2}}\right) \alpha,
$$

[17], where $\mathrm{u}$ is the axial velocity, $\mathrm{r}$ is the radial location in the cylinder cross-section, $\mathrm{t}$ is time, $\mathrm{i}$ is $\sqrt{-1}, \mathrm{k}$ is the amplitude of the driving pressure gradient, $\mathrm{R}$ is the pipe radius, $\mu$ is dynamic viscosity, $\alpha$ is the Womersley parameter, $\mathrm{J}_{0}$ is the Bessel function of first kind and order zero and $\omega$ is the angular frequency of the pressure gradient. By integrating Eq. (2), the mass flow rate is obtained,

$$
\dot{m}(t)=\frac{k B}{\alpha^{2}} e^{i \omega t}, \quad B=\frac{i \pi R^{4}}{v}\left(1-\frac{2 J_{1}(A)}{A J_{0}(A)}\right),
$$

where $\mathrm{J}_{1}$ is the Bessel function of first kind and order. As the pressure gradient driving the flow in the aqueduct of Sylvius is periodic, it can be described using a Fourier series, so that from Eqs. $(1,3)$ and Poisseuille's law [18] the mass flow rate can be expressed as

$$
\dot{m}(t)=\frac{-k_{0} \pi R^{4}}{8 v}+\frac{1}{\alpha^{2}} \sum_{n=1}^{\infty}\left(k_{2 n-1} \operatorname{Re}\left(B e^{i \omega t}\right)+k_{2 n} \operatorname{Im}\left(B e^{i \omega t}\right)\right),
$$

where Re and Im designate real and imaginary parts, respectively. This mass flow rate has to be equal to the one obtained by integrating the velocity field measured with velocimetric MR. Using weighted least-squares error minimization, the coefficients $\mathrm{k}$ were calculated and, hence, the velocity profile at the inferior end of the aqueduct was determined.

\subsection{Reconstruction of the Flow Field}

For the flow field reconstruction, the CSF was regarded as an incompressible Newtonian fluid with the same material properties as water at $37^{\circ}$ Celsius $[6,19]$. Three sets of non-uniform, unstructured computational grids, respectively consisting of about 257,000, 558,000 and 811,000 tetrahedral elements were used to carry out the finite-volume calculations with the CFD code Fluent (Fluent Inc., Lebanon, NH) using an implicit Algebraic Multigrid Scheme with PISO pressure correction [20].

Grid independence tests showed that the intermediate grid was fine enough to resolve all of the flow features accurately. Time step independence tests carried out with time step sizes of $\mathrm{T} / 100, \mathrm{~T} / 1,000$ and $\mathrm{T} / 10,000$, where $\mathrm{T}$ is the length of one 
period, showed that a time step size of T/1,000 was necessary to adequately model the flow. A period independence study demonstrated that three periods were necessary for the transients originating from the zero velocity and zero pressure initial condition to abate.

\section{Results}

Figure 3 shows contour lines of velocities perpendicular to the corresponding section planes, whose positions are shown in Figure 2, right side. The states at 0\%, 25\%, 50\% and at $75 \%$ of the duration of the cycle are depicted. Initially, the cerebrospinal fluid flows in cranial direction (see Fig. 2, left). A jet directed towards the foramina of Monro exits the aqueduct of Sylvius with a peak velocity of slightly less than $12 \mathrm{~cm} / \mathrm{s}$. Before flow reversal occurs at around $19 \%$ of the cycle, an area of recirculation develops above the jet, with its center at about the height of the recessus pinealis. After flow reversal, the recirculation splits the path of CSF flowing in caudal direction from the foramina of Monro into two: one part of the CSF is deflected in cranial direction, flows past the recirculation along the top wall of the ventricle and then in caudal direction towards the aqueduct, without entering the recessus suprapinealis. The second CSF stream is only slightly disturbed and flows caudal of the area of recirculation almost straight into the aqueduct. Before the second flow reversal occurs at about $75 \%$, this first recirculation has already dissipated and a second one is formed approximately half way between and caudal to the foramina of Monro and the adhesio interthalamica. By the time the cycle is $95 \%$ through, this second, bigger, recirculation has also dissipated.

The flow velocities in the treated domain are in general below $1 \mathrm{~cm} / \mathrm{s}$. Exceptions are the flow in the aqueduct with peak values of approximately $15 \mathrm{~cm} / \mathrm{s}$ at the narrowest cross-sections, the jet emerging from the aqueduct and the foramina of Monro, where peak velocity values of slightly above $2 \mathrm{~cm} / \mathrm{s}$ are reached. Maximum absolute velocities below $0.1 \mathrm{~cm} / \mathrm{s}$ are observed in all of the recessi.
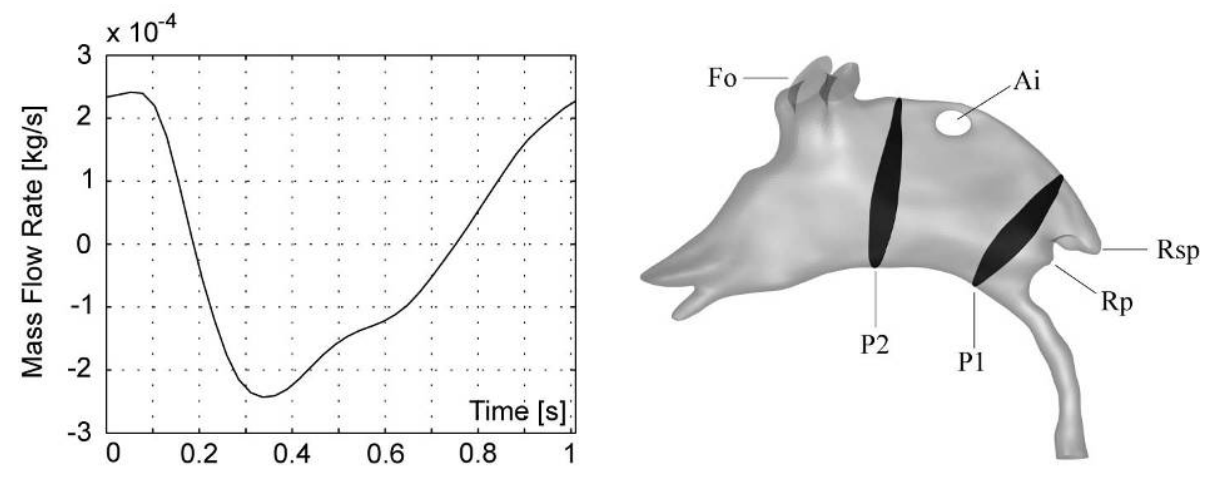

Fig. 2. Left: Mass flow rate through the inferior end of the aqueduct of Sylvius. Positive values indicate cranial direction. Right: Flow domain on which the finite-volume calculations were performed. P1 and P2 are section planes referred to in Fig. 3. Fo: foramina of Monro, Rp: recessus pinealis, Rsp: recessus suprapinealis, Ai: adhesio interthalamica. 

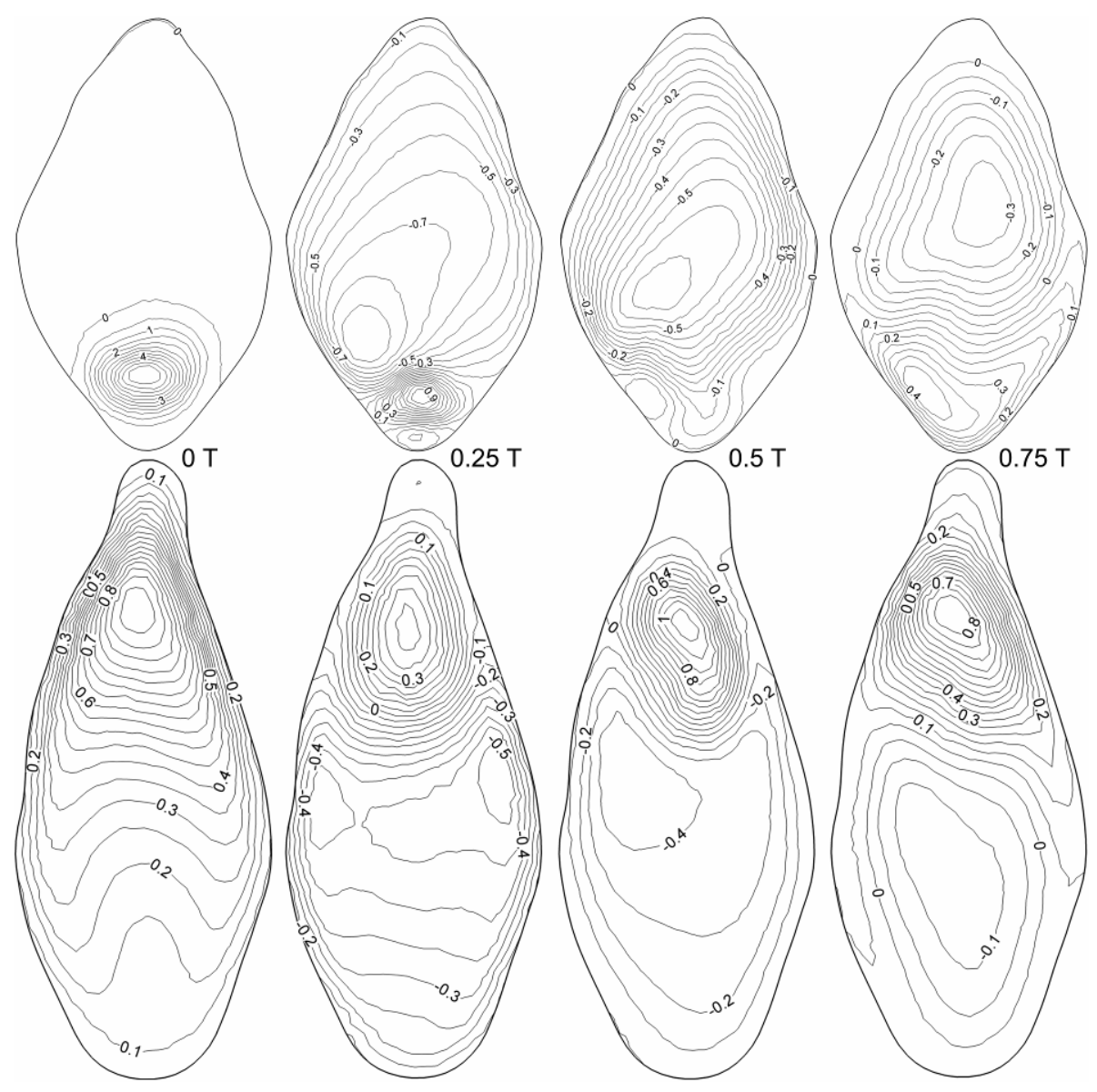

Fig. 3. Top row: Iso-contours of normal velocities in $\mathrm{cm} / \mathrm{s}$ in the section plane P1 (see Fig. 2, right) at $0 \%, 25 \%, 50 \%$ and $75 \%$ of the period $\mathrm{T}$ (Fig. 2, left). Positive values indicate cranial direction. Bottom row: Normal velocities in the plane P2.

\section{Discussion}

The basis of the simulation of cerebrospinal fluid flow in the third ventricle and the aqueduct of Sylvius as presented above are MRI scans of an individual. Therefore, the accuracy of the simulation is first and foremost governed by the accuracy of the MRI data acquisition. Due to the relatively high flow velocities occurring in the aqueduct (in the order of several $\mathrm{cm} / \mathrm{s}$ ) and the aqueduct's nearly cylindrical shape, the corresponding boundary condition can be reconstructed fairly accurately with a relative error well below $10 \%$. Anatomic MRI scans cannot resolve the detailed surface structures of the choroid plexus, which are in the order of micrometers. We believe that neglecting these structures in the third ventricle will have limited influence on the flow field, as they are located predominantly at the ventricle's top wall where the flow velocities are low. 
The motion of the brain tissue is responsible for the pulsatile flow of the cerebrospinal fluid. Hence, it is important to take it into account when reconstructing the CSF flow. MRI tagging can be used to capture the brain motion in feet-head direction (maximum amplitude $0.25 \mathrm{~mm}$ ). However, it will not capture the motion in the remaining directions which generally portray amplitudes less than $0.15 \mathrm{~mm}$. The velocity field specified at the inferior end of the aqueduct of Sylvius makes up for the lack of that data to a large extent. For clinical use, however, more advanced techniques will have to be used in order to take into account brain motion in every direction [21].

\section{Conclusions}

The resolution of current MRI scanners is not high enough to capture the fine details of the cerebrospinal fluid flow in the brain ventricular system. The use of idealized CFD simulations, on the other hand, will not capture patient-specific flow features that may be crucial to the outcome of the intervention. By relying on MRI measurements to capture the patient's anatomic structures and to provide boundary conditions for the computer simulations, these shortcomings can be eliminated. Precise knowledge of cerebrospinal fluid flow would allow for better planning of surgical interventions in those cases where the CSF space is involved. The prime example is shunt placement for the treatment of hydrocephalus and other conditions where CSF drainage or control is important.

We have shown that it is possible to combine anatomic, velocimetric and tagged MRI scans with CFD simulations to reconstruct the CSF flow field in the third brain ventricle and the aqueduct of Sylvius. The methods described herein can be applied without significant modifications to the entire ventricular system.

Acknowledgements. The financial support of the ETH Zurich Research Commission has made this research possible and is kindly acknowledged.

\section{References}

1. Davson, H., Segal, M.B.: Physiology of the CSF and Blood-Brain Barriers. CRC Press, Boca Raton (1996)

2. Agarwal, G.C., Berman, B.M., Stark, L.: A Lumped Parameter Model of the Cerebrospinal Fluid System. IEEE Transactions on Biomedical Engineering 16 (1969) 45-53

3. Jacobson, E.E., Fletcher, D.F., Morgan, M.K. et al.: Computer Modelling of the Cerebrospinal Fluid Flow Dynamics of Aqueduct Stenosis. Medical \& Biological Engineering \& Computing 37 (1999) 59-63

4. Loth, F., Yardimci, M.A., Alperin, N.: Hydrodynamic Modeling of Cerebrospinal Fluid Motion within the Spinal Cavity. ASME Journal of Biomechanical Engineering 123 (2001) 71-79

5. Fin, L., Grebe, R.: Three Dimensional Modeling of the Cerebrospinal Fluid Dynamics and Brain Interactions in the Aqueduct of Sylvius. Computer Methods in Biomechanics and Biomedical Engineering 6 (2003) 163-70 
6. Kurtcuoglu, V., Poulikakos, D., Ventikos, Y.: Computational Modeling of the Mechanical Behavior of the Cerebrospinal Fluid System. ASME Journal of Biomechanical Engineering 127 (2005) 264-269

7. Haacke, E.M., Brown, R.W., Thompson, M.R. et al.: Magnetic Resonance Imaging: Physical Principles and Sequence Design. John Wiley \& Sons, New York (1999)

8. Martinez, J.: Practical Quasi-Newton Methods for Solving Nonlinear Systems. Journal of Computational and Applied Mathematics 124 (2000) 97-121

9. Lanczos, C.: Applied Analysis. Pitman, London (1957)

10. Fischer, S.E., McKinnon, G.C., Maier, S.E. et al.: Improved Myocardial Tagging Contrast. Magnetic Resonance in Medicine 30 (1993) 191-200

11. Osman, N.F., Kerwin, W.S., McVeigh, E.R. et al.: Cardiac Motion Tracking using CINE Harmonic Phase (HARP) Magnetic Resonance Imaging. Magnetic Resonance in Medicine 42 (1999) 1048-60

12. Soellinger, M., Ryf, S., Kozerke, S. et al.: Brain Motion Measurements using CSPAMM. In 21th Annual Scientific Meeting of the ESMRBM, Copenhagen (2004)

13. Stahlberg, F., Mogelvang, J., Thomsen, C. et al.: A method for MR Quantification of Flow Velocities in Blood and CSF using Interleaved Gradient-Echo Pulse Sequences. Magnetic Resonance Imaging 7 (1989) 655-67

14. Womersley, J.R.: Oscillatory Motion of a Viscous Liquid in a Thin-Walled Elastic Tube. 1. The Linear Approximation for Long Waves. Philosophical Magazine 46 (1955) 199-221

15. Friedmann, M., Gillis, J., Liron, N.: Laminar Flow in a Pipe at Low and Moderate Reynolds Numbers. Applied Scientific Research 19 (1968) 426-438

16. He, X.Y., Ku, D.N.: Unsteady Entrance Flow Development in a Straight Tube. ASME Journal of Biomechanical Engineering 116 (1994) 355-360

17. Zamir, M.: The Physics of Pulsatile Flow. Springer Verlag, New York (2000)

18. Wilcox, D.C.: Basic Fluid Mechanics. DCW Industries, La Cañada (1999)

19. Bloomfield, I.G., Johnston, I.H., Bilston, L.E.: Effects of Proteins, Blood Cells and Glucose on the Viscosity of Cerebrospinal Fluid. Pediatric Neurosurgery 28 (1998) 246-51

20. Issa, R.I.: Solution of the Implictly Discretised Fluid Flow Equations by Operator Splitting. Journal of Computational Physics 62 (1985) 40-65

21. Soellinger, M., Rutz, A., Kozerke, S. et al.: Direct Quantification of Brain Motion. In $43^{\text {rd }}$ Annual Meeting of the ASNR, Toronto (2005) 